\title{
Diseño e implementación de un software SCADA para el módulo de temperatura del CEMA
}

\begin{abstract}
RESUMEN
El presente artículo presenta los resultados del proyecto de investigación que tuvo como objetivo principal diseñar e implementar un software SCADA en el modulo de temperatura del CEMA (Centro de Manufactura Avanzada, de la UNMSM); al final del proyecto se desarrolló tecnología propia la cual se materializó en un programa de software SCADA que será de utilidad para las clases de laboratorio del curso de Operaciones Unitarias así como un manual de laboratorio que pueda guiar al alumno en la elaboración de su propio programa o software.
\end{abstract}

Palabras clave: SCADA, sistema de control, temperatura, módulo.

DESING AND IMPLEMENTATION OF A SCADA SOFTWARE TO THE CEMA TEMPERATURE MODULE

\section{ABSTRACT}

The article shows the results of the research project that had like main objective design and implements a SCADA software in the CEMA temperature module; at the end of the project we develop own technology that finished in a SCADA software will be used in the course Units Operations, as well as a laboratory guide to can help to the students to make their own software.

Key words: SCADA, control system, temperature, module

\section{INTRODUCCIÓN}

La presente investigación se realizó en el módulo de control de temperatura del Centro de Manufactura Avanzada de la Facultad de Ingeniería Industrial de la UNMSM (CEMA). El resultado ha sido un programa software SCADA para control de temperatura desarrollado en el lenguaje $G$ del software LabView.

Para un mejor entendimiento es necesario indicar que el módulo de control de temperatura del CEMA trabaja con un software SCADA desarrollado por la empresa proveedora. Con el sistema de conexión actual a dicho módulo, el software muchas veces pierde comunicación con el PLC, lo cual produce una demora en la corrección, porque (entre muchos otros motivos) la programación no es propia, esto origina retrasos en el aula de clase y dependencia del proveedor. Por lo tanto, surgen las siguientes interrogantes problemáticas:

¿Cómo optimizar el funcionamiento del software SCADA del módulo de temperatura?

¿Cómo generar tecnología propia que permita el control del módulo de temperatura?

¿Es factible que esta tecnología pueda ser aplicada en una empresa con procesos que involucren control de temperatura?

De lo anteriormente mencionado, surgen los siguientes objetivos:

Optimizar mediante un software propio, el funcionamiento del módulo de temperatura.

Generar tecnología propia y su correspondiente documentación, mediante la programación de un programa de software SCADA en Labview para el módulo de temperatura.

Simular el funcionamiento en una empresa del software SCADA propio.

En cuanto al módulo de temperatura, el cual es de interés para la investigación, cuenta con un PLC Allen Bradley - Micrologix 1200 , el cual está conectado mediante un cable RS232 a una computadora para el monitoreo de la temperatura.

1 Magíster en Ingeniería Industrial. Docente Auxiliar del Departamento de Diseño y Tecnología Industrial. UNMSM. E-mail: achungp@industrial.unmsm.pe

2 Magister en Informática, Docente Principal del DAISI, Facultad de Ingeniería Industrial UNMSM. E-mail: eruizl@industrial.unmsm.pe 
Asimismo, cuenta con un sensor de temperatura que está conectado al PLC anteriormente mencionado, el actuador es una resistencia acoplada a un relay.

El software SCADA utilizado se conecta al controlador mediante el protocolo MODBUS.

\section{SOFTWARE SCADA}

SCADA significa: Control Supervisor y Adquisición de Datos; por lo tanto un software SCADA es aquel que puede realizar estas actividades dentro un sistema de control de procesos.

Un software SCADA se comunica con el usuario a través de una interfaz hombre máquina ( $\mathrm{HMI}$, Human Machine Interface); el software a utilizarse para la investigación será el cual no es un software SCADA sino un software de programación para software SCADA.

Según lo definido anteriormente, un sistema de control de procesos debe (entre otras funciones) interactuar con el usuario, esta interacción se va a realizar mediante el software SCADA va a permitir monitorear el proceso y a la vez asignar los valores respectivos a la variable Set Point.

\section{PROTOCOLO DE COMUNICACIONES}

El protocolo de comunicaciones es el lenguaje con el cual se comunican diversos instrumentos entre sí y con el controlador. El protocolo se divide en tramas, las cuales llevan la información entre los instrumentos.
Asimismo, las tramas se dividen como mínimo en cabecera, datos y cola; cada uno contiene información básica que alimenta el sistema; la Figura N. ${ }^{\circ} 1$ ilustra el tema.

La cabecera contiene información de identificación del protocolo, los datos contienen la información del esclavo, la operación a realizar y los datos necesarios así como el código de error y la cola es información complementaria.

\section{LENGUAJE G}

Para la realización de la investigación se procedió a la programación en lenguaje $\mathrm{G}$ de LabView; este lenguaje tiene la característica de trabajar con objetos gráficos los cuales se enlazan para formar estructuras o programas. Labview fue creado por National Instruments en 1976 para funcionar en maquinas MAC, en 1986 salió al mercado y actualmente está disponible para plataformas Windows, UNIX, LINUX, y por supuesto MAC.

El lenguaje $G$ al ser un lenguaje gráfico es de fácil compresión y además permite al programador visualizar directamente en el programa el algoritmo que está utilizando, lo cual no se puede lograr con otros lenguajes que trabajan con instrucciones escritas.

\section{RESULTADOS OBTENIDOS}

La Figura N. ${ }^{\circ} 2$ muestra la programación del software SCADA hecho en el presente proyecto.

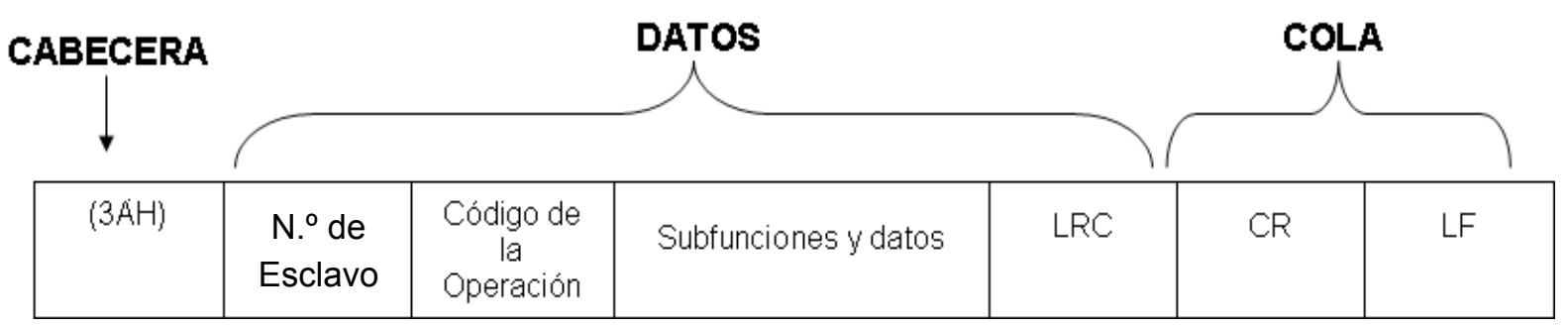

Fuente: Elaboración propia con datos de http://www.dte.upct.es/personal/manuel.jimenez/docencia/GD6_Comunic_Ind/pdfs/Tema\%207.pdf Visitada el 01/12/2010

Figura N. ${ }^{\circ}$ 1. Detalle de una trama. 


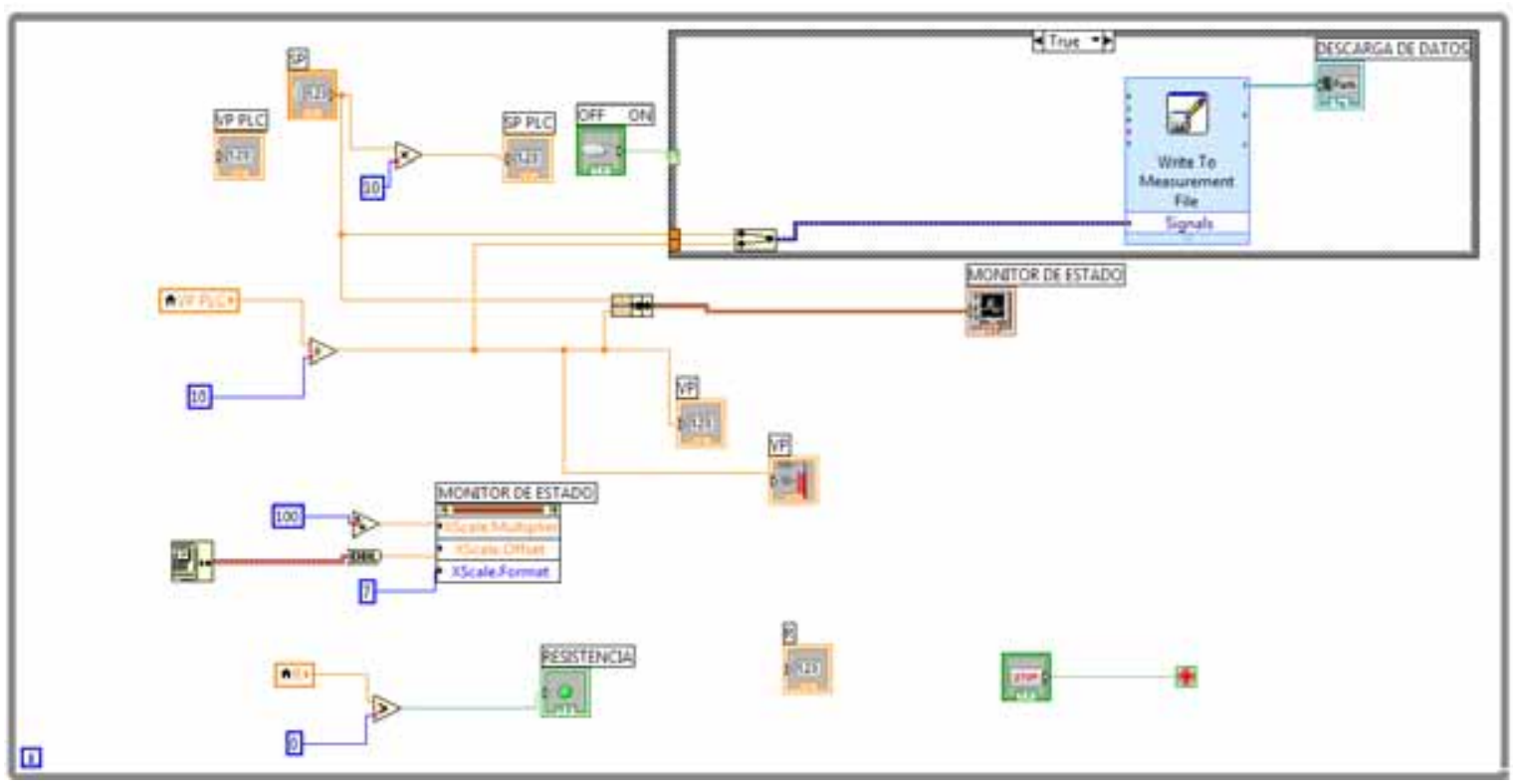

Figura N.ํ․ 2. Programación en lenguaje G.

\section{El algoritmo en pseudocódigo es el siguiente:}

Inicio

Objetivo: Controlar la temperatura del proceso

\section{Objetos:}

VP_PLC captura el valor de la variable de proceso del PLC

SP es el Set Point

SP_PLC captura el valor de SP y lo coloca en el PLC

$\mathrm{R}$ captura dato obtenido por el PLC de la resistencia

Variables:

VP Captura valor del objeto VP_PLC

$R$ Captura valor del objeto $R$

Objetos gráficos:

VP variable de proceso expresado en escala grafica

Resistencia

Mientras botón STP = true hacer

Leer valor SP de parte del usuario

Leer valor del PLC

Hacer $\mathrm{SP} \leftarrow \mathrm{SP}^{*} 10$

Escribir SP en el objeto SP PLC

Escribir SP_PLC en el sector 40002 del PLC

Escribir dato SP a la sub rutina "Write To Measurement File"

Escribir SP al Bundle

Almacenar en VP el valor del objeto VP_PLC

Hacer VP $\leftarrow$ VP / 10

Escribir VP a la sub rutina "Write To Measurement File"

Escribir VP al objeto grafico VP

Enviar VP al Bundle

Bundle escribe los dos valores a la "Wave Form Chart"

Objeto R captura el valor del sector 40009 del PLC el cual contiene información ON/OFF de la Resistencia.

Variable $R$ captura el valor del objeto $R$

If $\mathrm{R}>0$ Then

Resistencia $\leftarrow 1$

\section{Else}

Resistencia $\leftarrow 0$

If objeto OFF_ON $=$ true

"Write To Measurement File" la información al archivo en Excel

Fin Mientras

Fin 
A continuación se detalla un poco más los objetos el programa:

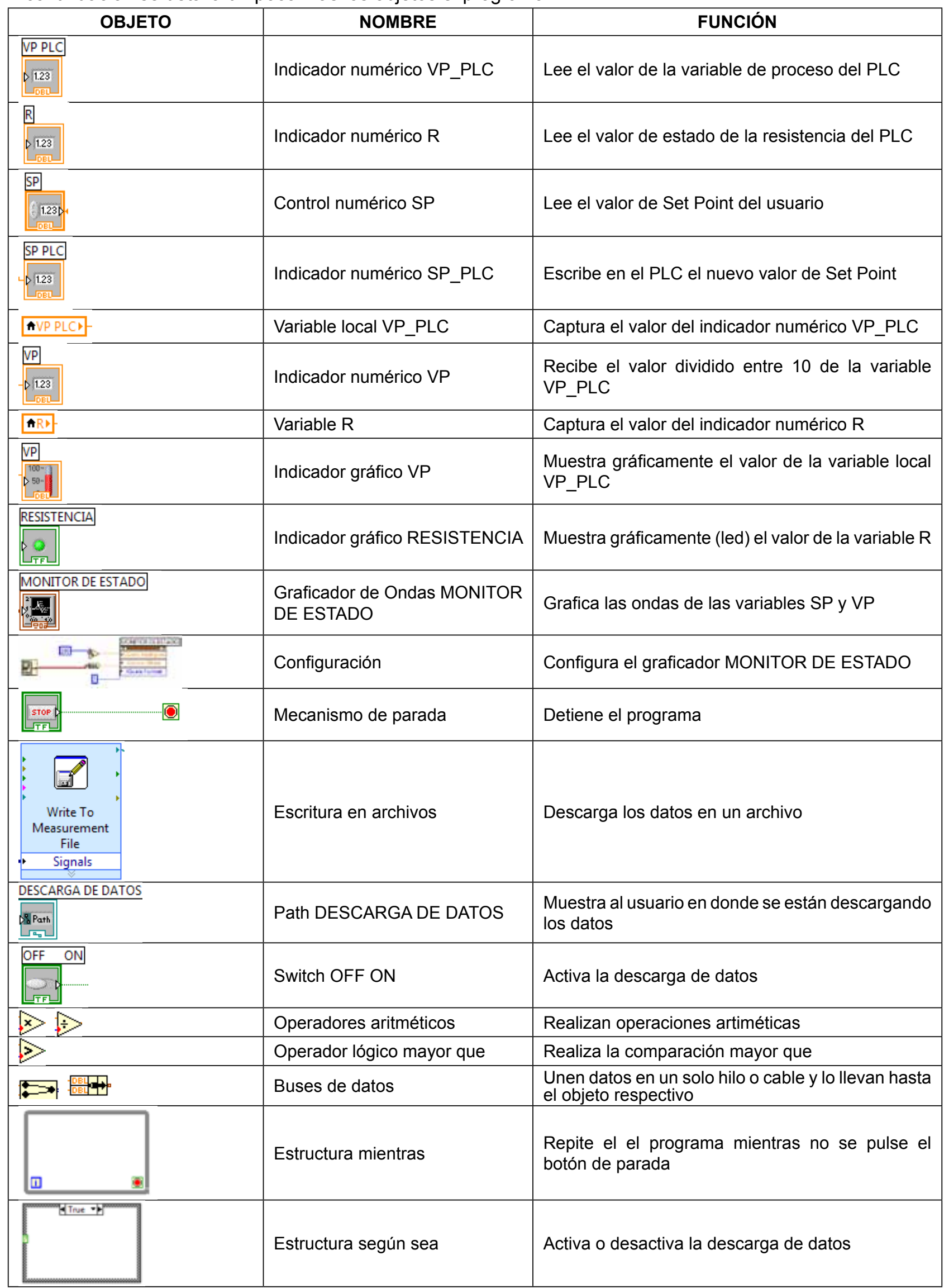




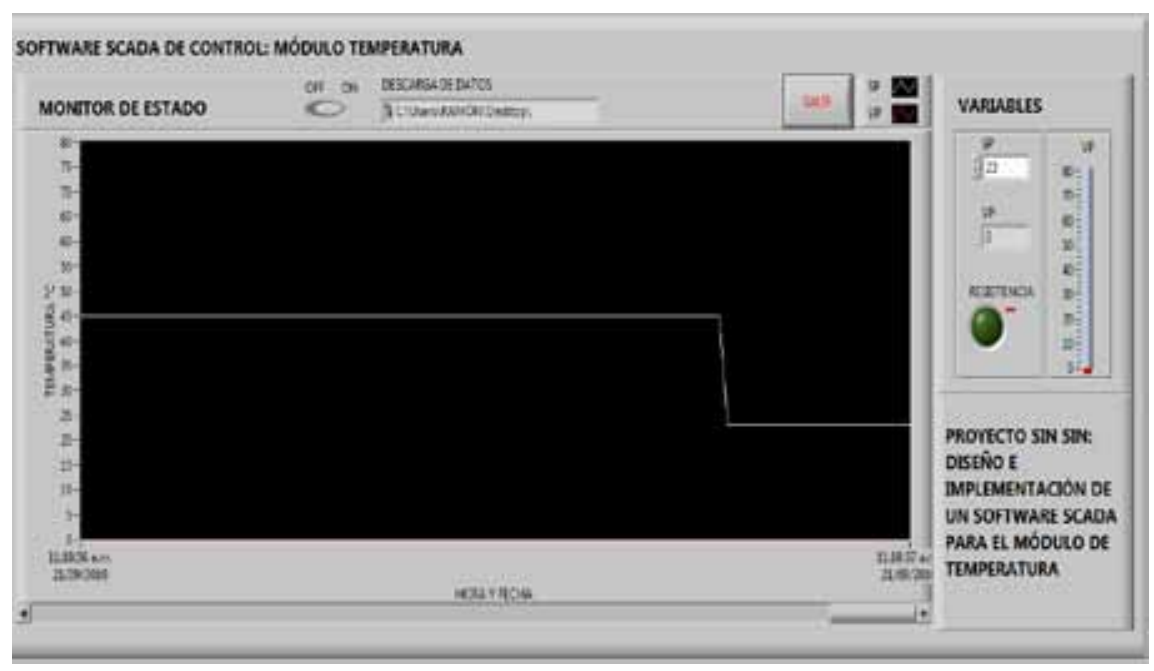

Figura $\mathbf{N}^{\circ}{ }^{3}$ 3. Interfaz de usuario.

La interfaz de usuario se presenta en la Figura $N .^{\circ} 3$ :

Como se puede apreciar en la figura anterior, se diseñó una interfaz que sea fácil de trabajar para el usuario; en dicha interfaz se puede identificar lo siguiente:

- Una entrada para que el usuario ingrese el valor de Set Point (SP), el cual podrá ver tanto como número como por una curva.

- Tres formas de mostrar la variable de proceso (VP), mediante un indicador numérico, por una curva y a través de un indicador gráfico (termómetro).

- Un led que se enciende cuando la resistencia está activada.

- Una palanca (ON - OFF) para iniciar la grabación de datos en la ruta indicada.

- Un botón para salir del programa.

\section{DISCUSIÓN DE RESULTADOS}

Los resultados obtenidos a la fecha finalización del proyecto permiten plantear el reemplazo del software SCADA que se viene usando en el CEMA por este nuevo software de control de procesos.

Al diseñar y programar el software de control, el docente tendría pleno control del mismo, lo cual es una ventaja para realizar actualizaciones y también por si se presentan casos de funcionamiento inadecuado.

Finalmente, se ha logrado la generación de tecnología propia, lo cual llevará a la posterior transferencia de la misma a los alumnos en el CEMA.

\section{CONCLUSIONES}

La programación gráfica presenta una estructura de fácil entendimiento inclusive que la programación tradicional escrita.

Al diseñar e implementar un determinado programa o software, las tareas de mantenimiento y actualización se pueden hacer en forma oportuna, casi independiente del proveedor y ajustado a las necesidades de la organización.

La capacitación del componente humano en nuevas tecnologías es un factor básico para el desarrollo de toda organización.

La transferencia tecnológica debe constituirse como el corolario de todo proceso de mejora, ya que esto ayudará a que el avance se preserve y sea superado en el futuro.

\section{REFERENCIAS BIBLIOGRÁFICAS}

[1] Chung, A., Godoy, M. (2009), Presentaciones de control de procesos, Facultad de Ingeniería Industrial UNMSM.

[2] Chung, A. (2009), Manual de Laboratorio de Operaciones Unitarias, UNMSM.

[3] Inducontrol S.A.C. (2007), Manual del Módulo de Control de Temperatura, Inducontrol S.A.C.

[4] Maloney, T. (2006) Electrónica Industrial Moderna. 5ta. Ed, Pearson, México.

[5] Sánchez, J. (2003), Control Avanzado de Procesos, 1era. Ed., Diaz de Santos, España.

[6] Richard, M Ronald, W. (2004). Principios Básicos de los Procesos Químicos, 3era. Ed., Limusa Wiley, México. 